\title{
Ultrastructure of marine snow. I. Transmission electron microscopy methodology
}

\author{
Gary G. Leppard ${ }^{1}$, Andreas Heissenberger ${ }^{2}$, Gerhard J. Herndl ${ }^{2, *}$ \\ 'Aquatic Ecosystem Protection Branch, National Water Research Institute, Environment Canada, Burlington, Ontario, \\ Canada L7R 4A6; and Department of Biology, McMaster University, Hamilton, Ontario, Canada L8S 4K1 \\ ${ }^{2}$ Institute of Zoology, University of Vienna, Althanstr. 14, A-1090 Vienna, Austria
}

\begin{abstract}
Four different approaches to determine the ultrastructure of amorphous aggregates and their principal components are evaluated for their ability to resolve structural components as small as $1 \mathrm{~nm}$. Based on extensive comparative analysis, the advantages and major drawbacks of the different methods are discussed. Based on experience with marine snow from the Adriatic Sea, it is concluded that only a combination of fixation and preservation treatments ensures optimal interpretation of the results. Specific emphasis is placed on the use of a hydrophilic resin (Nanoplast $\left.{ }^{(}\right)$) which allows fixation of highly hydrated polysaccharide fibrils in their original configuration and subsequent visualization on a nom scale
\end{abstract}

KEY WORDS: Marıne snow. Transmission electron microscopy Mucus · Colloids Polysaccharıdes Fibrils - Nanoplast ${ }^{(*)}$ Adriatıc Sea

\section{INTRODUCTION}

Marine snow is a collective term for suspended, fragile, amorphous flocs with diameters of at least $0.5 \mathrm{~mm}$ (Suzuki \& Kato 1953, Alldredge \& Silver 1988). These heterogeneous flocs are basically aggregations of mucilage, organisms (both living and dead) and detritus. The specific physical and chemical properties of the mucilaginous matrix depend upon the species of the secreting organisms (Percival \& McDowell 1967. Kenne \& Lindberg 1983, Painter 1983) and ultimately determine the overall structure of an aggregate and its microbial associations (Leppard 1995)

More than 100 different sugar species and sugar derivatives are present as monomeric constituents in polysaccharides (Lindberg 1981). The complexity of polysaccharides is additionally increased by the different possibilities of linkages (including branching linkages) and the variable molecular weight (up to millions of daltons) rithin a single family of polysaccharide spo-

- Addressee for correspondence.

E-mail: gerhard.herndl@univie.ac.at cies (Aspinall 1982); furthermore, a large variation in the secondary structure and molecular associations adds to the complexity of the polysaccharides (Rees et al. 1982, Kloareg \& Quatrano 1988). On the other hand, some distinct features facilitate characterization of these large molecules: (1) many important polysaccharides contain only a small number of monomeric species (Percival \& McDowell 1967); (2) they contain repetitive features in their primary chemical structure which permit classification in lieu of complete analyses of the chemical structure (Aspinall 1983, Whitfield 1988); (3) many important polysaccharides contain carboxyl-and/or sulfate-functional groups, thus giving the macromolecule an acidic character; (4) many form aggregates, including heteroaggregates, which give rise to the distinctive fibrillar structures known as fibrils or fibrillar extracellular polymeric substances (Leppard 1992a, b, 1993)

Fibrils have been incidentally visualized in marine snow in the past by the prosont authors and by others, anecdotal evidence suggests that fibrils could very well be the principal component of the polysaccharide matrix in marine snow, connecting with each other 
predominantly via cationic bridging (van Boekel 1992, Decho \& Hennd 1995) to provide a discrete physical framework for microbial associations within the matrix. Because of their small size ( 3 to $10 \mathrm{~nm}$ in diameter) individual fibrils cannot be seen by optical microscopy, although large aggregations of them can be detected as an 'amorphous' material stained by basic dyes. Fibril aggregations are physically unstable (Leppard et al. 1990) and require high resolution electron-optics for visualization. For physical characterization of these delicate structures in a native state, inordinate attention to sample handling and sample stabilization techniques is required (Leppard et al. 1990, Perret et al. 1991, Filella et al. 1993).

As a result of methodological problems with sample stabilization, 3-dimensonal arrays of fibrils have not been amenable to high resolution analyses in marine snow, with extraction and dehydration-induced shrinkage causing the most serious artifacts. A multi-method protocol which allows one to detect, assess and then systematically minimize artifacts of preservation has been suggested as a solution to such methodological problems by the International Union of Pure and Applied Chemistry (IUPAC) (Leppard 1992a). Our research goal is therefore to visualize marine snow particles, at 1 to $3 \mathrm{~nm}$ resolution, as miniature ecosystems whose natural associations of microbes and colloidal materials might provide new insights into the ecology of marine snow.

In this study we describe a new method to fix polysaccharide fibrils for later examination by transmission electron microscopy (TEM). This new preparatory technique was originally developed for freshwater samples (Leppard 1992a, Liss et al. in press) and uses a hydrophilic resin to embed the samples in order to fix the highly hydrated polysaccharides in their native positions with their natural associations. The hydrophilic resin of choice, Nanoplast ${ }^{\circledR}$, is the only one demonstrated thus far to provide optimal qualities for the embedding of hydrated fibrils (Frosch \& Westphal 1989. Perret et al. 1991, Liss et al. in press). This embedding technique is applied in combination with several different approaches in a correlative manner (Leppard 1992b) to further prepare the samples for investigation under TEM. Considerable attention is paid to reduction of sample perturbation and cytological artifacts. Using this new technique we are attempting to visualize all the fibrils which contribute to the structure of the marine snow matrix in their true 3dimensional forms. By minimizing shrinkage and loss of fibrils during cytological processing, we are also attempting to reveal the level of porosity of the matrix. Sampling along a trophic gradient was used to characterize and assess variation in the ultrastructural morphology of the so-called 'amorphous' matrix.

\section{MATERIALS AND METHODS}

Study site and sampling. Marine snow samples were collected along a trophic gradient across the northern Adriatic Sea in July 1993 (for the location of the sampling stations see Karner et al. 1992). Samples were taken from various depth layers with rinsed Niskin bottles as well as by SCUBA divers with handmade syringes as described in Herndl \& Peduzzi (1988). On board the RV 'Vila Velebita', marine snow from each sampling depth was collected with a Pasteur pipette with a minimum of ambient water and transferred in aliquot portions into BEEM capsules for later transmission electron microscopic (TEM) analysis; 4 different treatments were used in order to overcome specific artifacts inherent in each method (see detailed description below).

Transmission electron microscopy. Instrumentation: Ultrathin sections of embedded samples, mounted on electron microscope grids, were examined with a JEOL 1200 EXII TEMSCAN scanning-transmission electron microscope (STEM) operating at $80 \mathrm{keV}$ in transmission mode. For identification of mineral colloids embedded in or associated with marine snow, ultrathin sections were analyzed by energy-dispersive spectroscopy (EDS) (Chandler 1977) using the same STEM instrument equipped with a Princeton Gamma Tech Si(Li) X-ray detector (horizontal entry type used in conjunction with a PGT Imix multi-channel analyzer featuring a SUN 3/60 Unix workstation). Analyses of element composition by EDS for elements of $Z$ (atomic number) $\geq 11$ were made over counting periods adjusted to minimize sample decomposition. Morphological analysis was confined to exposure times which precluded sample alteration during documentation by photography (generally $2 \mathrm{~s}$ of exposure time).

Preparation techniques: To prevent storage artifacts (Perret et al. 1991, Leppard 1992a) marine snow samples were directly immersed into the stabilizing solutions for TEM. As outlined in Fig. 1, 4 different treatments (5 replicates each) were applied. Glutaraldehyde-based fixatives provide good to excellent preservation of cell contents but they frequently do not stabilize the mucilaginous matrix. The glutaraldehyde/ ruthenium red fixative preserves cells and mucilage equally well but often yields a grainy image which hinders documentation at high magnification (Luft 1971, Cagle et al. 1972, Leppard et al. 1977). The Nanoplast ${ }^{(B)}$ serves as both fixative and water-miscible embedding resin, but does not preserve cell contents well; however, it preserves, extracellular structures, many cell secretions and colloids in their hydrated form (Frosch \& Westphal 1989, Perret et al. 1991, Liss et al. in press). Nanoplast ${ }^{\circledR}$ preparations allow en bloc staining (Mavrocordatos et al. 1994) of small mucilagi- 


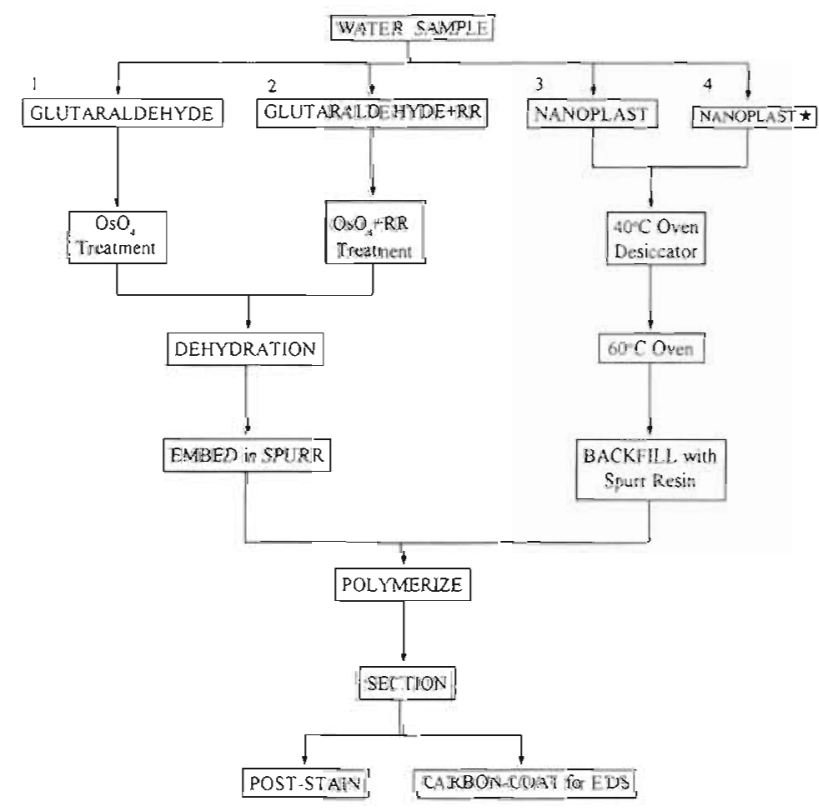

Fig. 1. Flow chart on the preparation of marine snow for TEM; 4 different preparatory treatments were performed; a protocol was developed to ensure maximum structural integnty and high resolution. ( ) uranyl acetate (or other en bloc stains); RR: ruthenum red; EDS: energy-dispersive spectroscopy. Adapted from Liss et al. (in press)

nous particles, which, potentially, facilitates detection of marine snow in the resin blocks prior to sectioning.

The glutaraldehyde-based primary fixative (Fig. 1) consisted of $2.6 \%$ EM-grade glutaraldehyde in $67 \mathrm{mmol}$ $\mathrm{I}^{-1} \mathrm{Na}$-cacodylate-HCl buffer set at pH 7.0 (Hayat 1981) and applied at ambient temperature. This solution was added $2: 1(\mathrm{v} / \mathrm{v})$ to the marine snow sample, mixed gently and allowed to sit for at least $2 \mathrm{~h}$. Thereafter, for washing in buffer, the samples were transferred into Eppendorf centrifuge tubes and centrifuged for 5 min at $1500 \times g$. The supernatant was removed with a disposable glass pipet and replaced with the same volume of the diluted $\mathrm{Na}$-cacodylate- $\mathrm{HCl}$ buffer (1:1 with distilled water). After $15 \mathrm{~min}$, the samples were centrifuged again, the supernatant was replaced by adding a more diluted $\mathrm{Na}$-cacodylate- $\mathrm{HCl}$ buffer (1:4 with distilled water). After $15 \mathrm{~min}$, this procedure was repeated to yield a pellet which was added to the secondary fixative, a $1.33 \%$ solution of osmium tetroxide in $67 \mathrm{mmol} \mathrm{l}^{-1} \mathrm{Na}$-cacodylate- $\mathrm{HCl}$ buffer at $\mathrm{pH} 7.0$. The pellet was suspended in $1 \mathrm{ml}$ of cold $\left(4^{\circ} \mathrm{C}\right)$ secondary fixative and allowed to sit for $2 \mathrm{~h}$. Excess osmium tetroxide was removed after centrifugation and the supernatant replaced as doscribod above. Subscquently, marine snow was transferred to a standard hydrophobic resin (Spurr's epoxy resin; Spurr 1969). The pellet was never allowed to dry in the air.
The primary fixative containing both glutaraldehyde and ruthenium red consisted of $2.6 \%$ glutaraldehyde (EM grade) and $0.05 \%$ ruthenium red (EM grade) in the same buffer as described above (Burnison \& Leppard 1983, Strycek et al. 1992). The protocol was basically the same as described above except that the secondary fixative consisted of $1.33 \%$ osmium tetroxide and $0.05 \%$ ruthenium red in the cacodylate buffer. The mixing of the osmium tetroxide and the ruthenium red in the buffer must be done immediately prior to use because of its tendency to form coarse precipitates after several hours (Leppard unpubl. data).

Nanoplast FB101(B), a hydrophilic melamine resin (Bachhuber \& Frosch 1983) in its medium hardness formulation (R. Bachhuber, Chemikalien für Elektronenmikroskopie, Ulm, Germany), was used as a watermiscible fixative/embedding resin (Fig. 1). It was used principally as an embedding resin to permit analysis of the 3-dimensional arrangements of hydrated colloids and bacteria associated with the mucilaginous matrix of marine snow. Because of its primary use as an embedding agent it is described in detail in the following section, including its use in en bloc staining.

Dehydration and embedding: Fixation (chemical stabilization and preservation) of marine snow in the 2 glutaraldehyde-containing fixatives (Fig. 1) was followed, after post-fixation, by dehydration in an organic solvent which was miscible with the hydrophobic epoxy resin of choice (Spurr 1969). Gentle dehydration was achieved by a graded methanol series in distilled water $(20,40,70,85,95 \%, 3$ times in $100 \%$ methanol). During all transfers the pellet was always covered by liquid.

The pelleted marine snow was embedded by pipetting off the supernatant methanol and replacing it with $0.25 \mathrm{ml}$ of an epoxy/methanol solution (1:1), then the suspension (marine snow in diluted epoxy) was centrifuged at $3000 \times g$ for $5 \mathrm{~min}$ in an Eppendorf tube. About 5 marine snow particles were removed from the pellet in the Eppendorf tube with a disposable glass pipet and transferred, together with the ambient liquid, into a glass vial containing $0.8 \mathrm{ml}$ of pure epoxy in medium hard formulation (Spurr 1969). This procedure resulted in a layer of marine snow in diluted epoxy resin on top of the pure epoxy resin in each glass vial. The marine snow particles were allowed to settle slowly to the bottom of the glass tube (within $12 \mathrm{~h}$ ); while settling, they became infiltrated with full strength epoxy resin. Approximately $50 \mu \mathrm{l}$ of particles including epoxy was removed from the bottom of the glass vial and then transferred to a BEEM capsule (type 00 large with conical tip). A pre-polymerization step of heating at $70^{\circ} \mathrm{C}$ for 3 h made the epoxy sufficiently viscous that the marine snow was held in the tip of the BEEM capsule after filling the capsule with additional epoxy; finally the epoxy was polymerized at 
$70^{\circ} \mathrm{C}$ for $7 \mathrm{~h}$. The staining techniques developed for this hydrophobic resin work well (Lewis \& Knight 1977. Leppard et al. 1990, Leppard 1992a); the 3-dimensional arrangement of the colloidal material embedded, however, can be altered due to centrifugation and dehydration, factors which can be avoided by the use of hydrophilic Nanoplast resin.

Samples $(4 \mu \mathrm{l})$ of marine snow were directly transferred from the sampling device (Niskin bottle or syringe as described above) into a BEEM capsule filled with $50 \mu \mathrm{l}$ of freshly prepared Nanoplast (Perret et al. 1991): to initiate polymerization of the Nanoplast ${ }^{(B)}$, the BEEM capsules were placed into an oven set at $40^{\circ} \mathrm{C}$ for $2 \mathrm{~d}$ in the presence of a desiccant and, thereafter, without a desiccant at $60^{\circ} \mathrm{C}$ for another $2 \mathrm{~d}$. This procedure ensures optimal properties for ultrathin sectioning (Frosch \& Westphal 1989) with a resolution limit of about $1 \mathrm{~nm}$. During polymerization, no perturbation by turbulence, compression or dehydration changes the native associations of the colloidal fibrils of the marine snow particles. As heating proceeds, the water-soluble hexamethylol-melamine-methyl-ether monomers penetrate the pore structure of the marine snow matrix to displace bulk water. These monomers polycondensate with each other, resulting in a macromolecular matrix which is less and less soluble in water as polymerization continues; thus the water becomes replaced by a plastic matrix which ties all colloid systems (e.g. fibrils, fibril-cell associations, fibril-mineral associations) together in their native 3-dimensional arrangement before loss of structural water can create dehydration artifacts (e.g. shrinkage).

Because of the current limitation on using selective stains for visualizing structures rich in organic carbon embedded in Nanoplast ${ }^{\circledR}$, the examination of ultrathin sections (50 to $70 \mathrm{~nm}$ ) for organic-rich structures (bacteria and mucilage) is very time consuming and thus costly. Applying an 'electron-opaque' stain to the Nanoplast ${ }^{\circledR}$ prior to addition of the sample leads to more readily recognizable organic-rich structures; we applied variable concentrations $(0.25,0.125$ and $0.06 \%, w / v)$ of uranyl acetate.

Sectioning and staining of sections: After polymerization all samples were sectioned identically. Ultrathin sections for morphological studies (50 to $70 \mathrm{~nm}$ ) and EDS analysis ( 80 to $100 \mathrm{~nm}$ ) were obtained from the polymerized resins by sectioning with a diamond knife mounted in an ultramicrotome (RMC Ultramicrotome MT-7). The sections were mounted on formvarcovered copper grids. The grids used for EDS were carbon-coated for stabilization. The Spurr sections were counter-stained with uranyl acetate (Lewis \& Knight 1977) and subsequently with lead citrate (Reynolds 1963). The Nanoplast ${ }^{\circledR}$ sections were counterstained with $1 \%$ aqueous uranyl acetate.
Optical microscopy. In order to obtain an overall view on the matrix of marine snow, we used a carbohydrate-selective stain and optical microscopy. Samples (1 or 2 aggregates with ambient water) were stained with a few drops of toluidine-blue solution $10.02 \%$ in distilled water) and mixed thoroughly. After 20 min the stained particles were covered with a cover slide and observed with a Nikon (Microphot-SA) optical microscope.

\section{RESULTS AND DISCUSSION}

During the investigation period, the marine snow was generally rather small, ranging from $0.5 \mathrm{~mm}$ to $2 \mathrm{~cm}$ in diameter, with no significant differences discernible between different stations or depth layers Light microscopical examination indicated that marine snow consisted of a polymeric matrix in which diatoms were the most abundant phytoplankton species.

\section{Assessment of the different preparations for TEM}

As shown in Fig. 1, 4 different procedures were performed to allow correlative TEM examination (Leppard 1992b) of marine snow from 6 sampling sites with 4 depth layers each. Additionally, samples taken with syringes were compared with those from Niskin bottles. Overall, the TEM investigation was based on 50 separate samples, each processed in 4 different ways with 5 replicates each and with as many as 1000 single abservations examined per replicate. The assessment focused on extraction and dehydration artifacts (Leppard 1993), including shrinkage. The possibility of storage artifact was excluded by immediate stabilization of the sample upon collection. On the basis of our direct observations of all the samples from several sampling sites and depths, the following generalizations on the performance of the different treatments can be drawn.

Treatment 1 yielded good to excellent images of the cytoplasm of intact cells for most cell types. The images of cell contents were of high quality at both high and low magnifications. The major drawbacks of this treatment were artifacts of extraction of matrix colloids and concomitant matrix shrinkage. More than $50 \%$ (often more than $80 \%$ ) of the extracellular matrix fibrils were extracted from the marine snow by the numerous exchanges of fluids. This extraction (removal of fibrils) led to an increase in the void volume of the matrix and a consequent collapse (recorded as shrinkage) during centrifugation steps. With the loss of polymer bridges between the subcomponents of a marine snow particle, a rearrangement of subcomponent associations became 
highly probable. An example of this preparation is demonstrated in Fig. 2; the cells and their contents are well preserved, but almost no fibrils are detectable. The mineral particles surrounding the cells have probably been compressed due to fibril loss and subsequent shrinkage of the matrix.

Treatment 2 (glutaraldehyde plus ruthenium red as the primary fixative) showed all the favorable qualities of Treatment 1 , with the exception that high magnification images (primary magnification of $50000 \times$ or higher) sometimes showed a grainy background of ruthenium red, which obscured the finest details. Comparing marine snow images obtained by optical microscopy (both native particles and particles stained for polyanionic matrices) with Treatment 2 in the TEM, we detected the same overall morphology of the most common marine snow particles. The characteristic features of marine snow readily recognizable were the numerical dominance and patchy distribution of bacterial cells, the bacteria-algae associations, the bacteria-debris associations and the presence of minerals in the matrix. Fig. $3 \mathrm{~A}$ shows the association of bacteria and mineral particles mediated by polysaccharide fibrils. In contrast to Treatment 1 (Fig. 2), fibril extraction was minimal, therefore the natural associations remained relatively undisturbed. Shrinkage went uncontrolled, however, as a result of centrifugation applied as an aid to fluid exchange steps. For the different types of marine snow examined in this study, the images obtained with Treatment 2 provided a useful approximation of the native state. Individual marine snow components and their associations were readily interpretable.

The real 3-dimensional arrangements of the polysaccharide fibrils can be obtained if observations of particles embedded according to Treatment 3 are used in correlation with those of particles embedded according to Treatments 1 and 2. Treatment 3 (standard Nanoplast ${ }^{\circledR}$ embedding) yielded images of minimal perturbation of the non-living components of marine snow (fibrils, organic debris, biogenic minerals) and the 3-dimensional arrangements of all structural components (Fig. 4). No solvent extractions or centrifugation steps were applied; thus we avoided extraction and rearrangement of the delicate and physically unstable, highly hydrated components. As can be seen in Fig. 4, this method allows high resolution (1 $\mathrm{nm})$ allowing the visualization of even the finest fibrils within the matrix, which are obscured by other techniques. This procedure allows therefore quantification

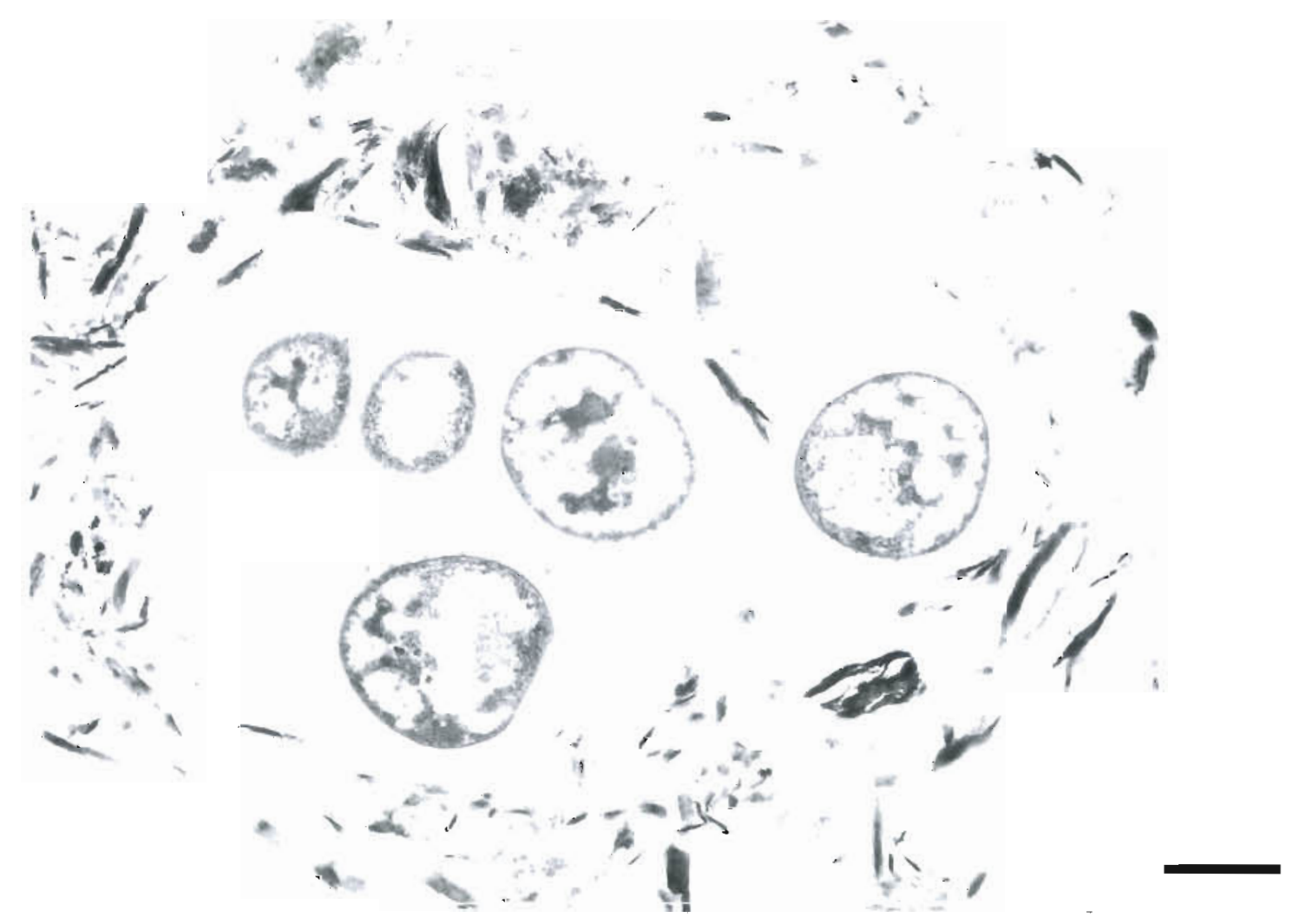

Fig. 2. TEM image of a characteristic section of a marine snow particle prepared by Treatment 1 It shows bacteria, mineral particles and biogenic debris as subcomponents. Intracellular details are well defined but fibrils are rare. Compare with a similar particle prepared by Treatment 2 (Fig. 3) which preserves matrix fibrils. Scale bar $=0.5 \mu \mathrm{m}$ 


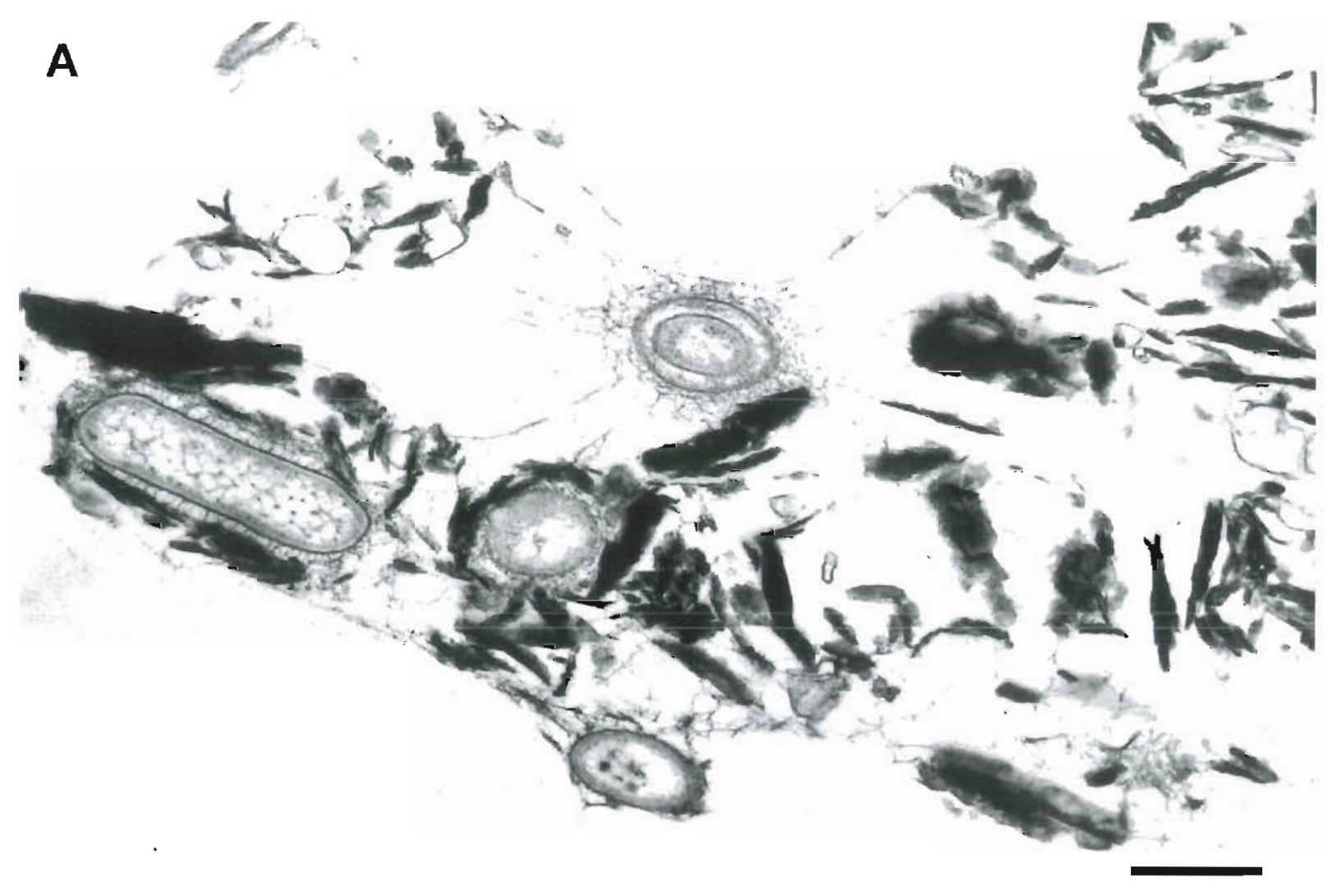

B
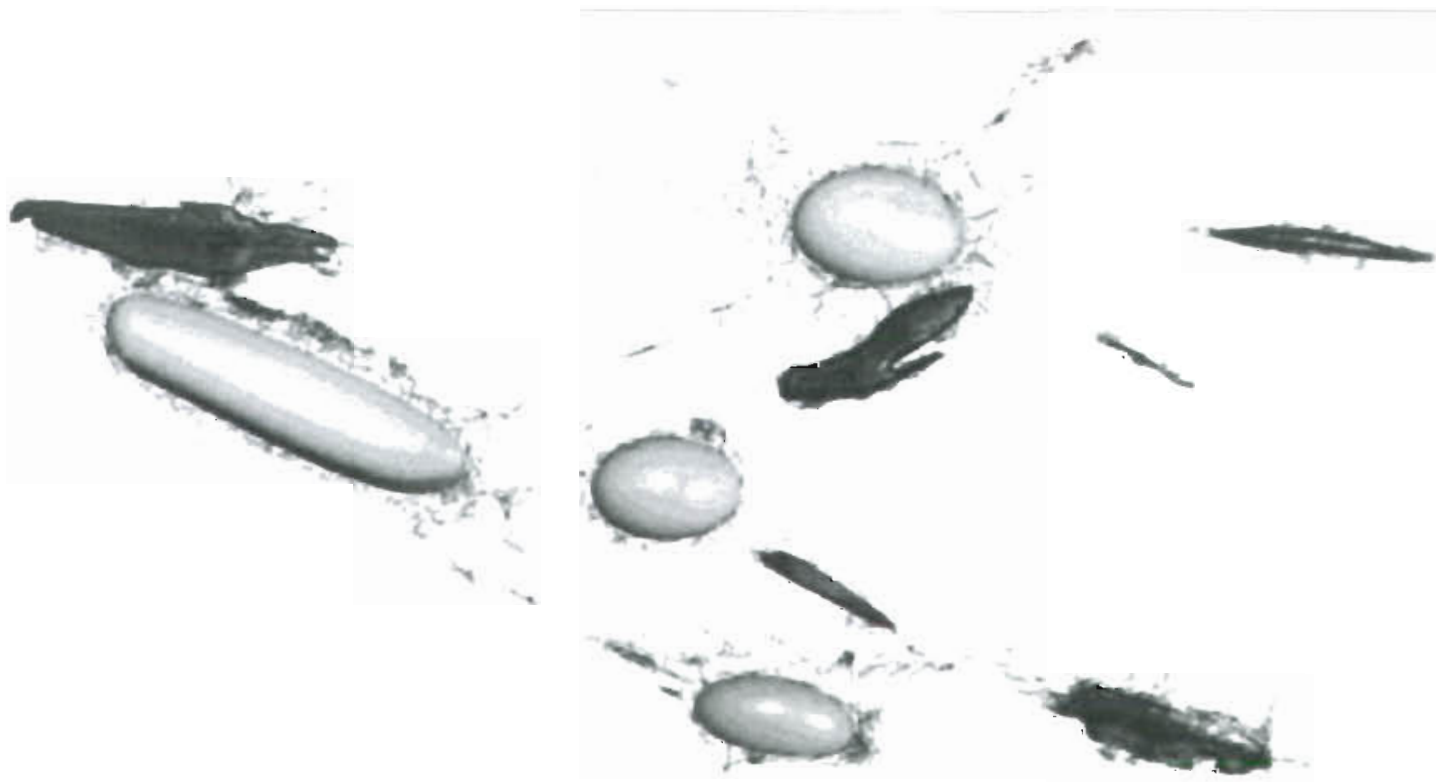

Fig. 3. Image of a characteristic section of a marine snow particle prepared by Treatment 2: (A) a TEM micrograph and (B) a computerized 3-dimensional reconstruction based on serial sections. Associations between bacteria and mineral particles and fibrils binding different kinds of subcomponents of the particle together are visible. Scale bar $=0.5 \mu \mathrm{m}$

(measurements of dimensions and shapes) of single fibrils with an independent verification possible by freeze-fracture methodology (Leppard 1992a, b). As is generally true for preparations in which Nanoplast ${ }^{-1}$ is used as both primary fixative and embedding medium, the internal details of cells were not well preserved. 


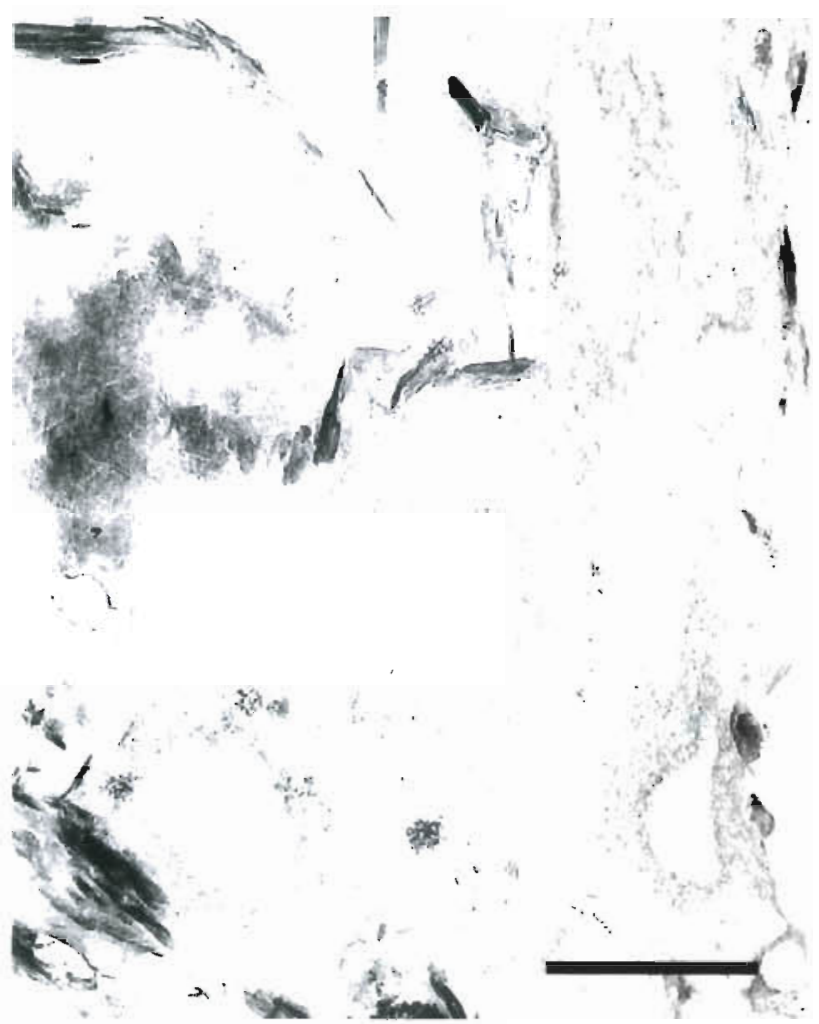

Fig. 4. TEM image of a fibril network embedded in Nanoplast $^{\ominus}($ Treatment 3$)$. Scale bar $=0.25 \mu \mathrm{m}$

Treatment 4 was not as effective for revealing marine fibrils as has been shown previously for freshwater samples (Mavrocordatos et al. 1994, Liss et al. in press). A 4 -fold dilution $(0.063 \%)$ of the uranyl acetate en bloc stain with distilled water, however, was found to be sufficient for low-grade en bloc staining. Other variations on en bloc staining are being pursued, with considerable promise.

Based on EDS analysis, the most common mineral particles were either silicon-rich or probable clay minerals which are rich in both silicon and aluminum (Fig 5). Many of the silicon-rich particles were fragments of diatom frustules and thus were biogenic silicates. As indicated by the spectrum in Fig. 5C, some particles contained potassium, magnesium and phosphorus. Also, traces of iron could be detected on some clays (Fig. 5B). Despite the overall relative density approximating unity, marine snow typically was rich in mineral particles and colloids.

\section{Correlative approach to image interpretation}

A composite picture can be constructed of the ultrastructure of marine snow by selectively using images produced from Treatments 1,2 and 3 (Table 1). Treat- ment 1 satisfies the highest quality standards of preservation/fixation for living cells. The exact formulation of the aldehyde primary fixative can be adjusted if specific details of certain cytoplasm components are of particular interest (Hayat 1981). Due to the low shrinkage in samples prepared by Treatment 2, one can readily visualize all major types of cells in their original proportions and those matrix components which bind small particles together For more detailed observations of the matrix, images from Treatment 3 provided the best results. The use of molecular markers and cytochemistry (Leppard et al. 1990) can be included in the protocols of Treatments 1 and 2, while measurements on the length and width of individual fibrils should be done on images obtained by Treatment 3 .
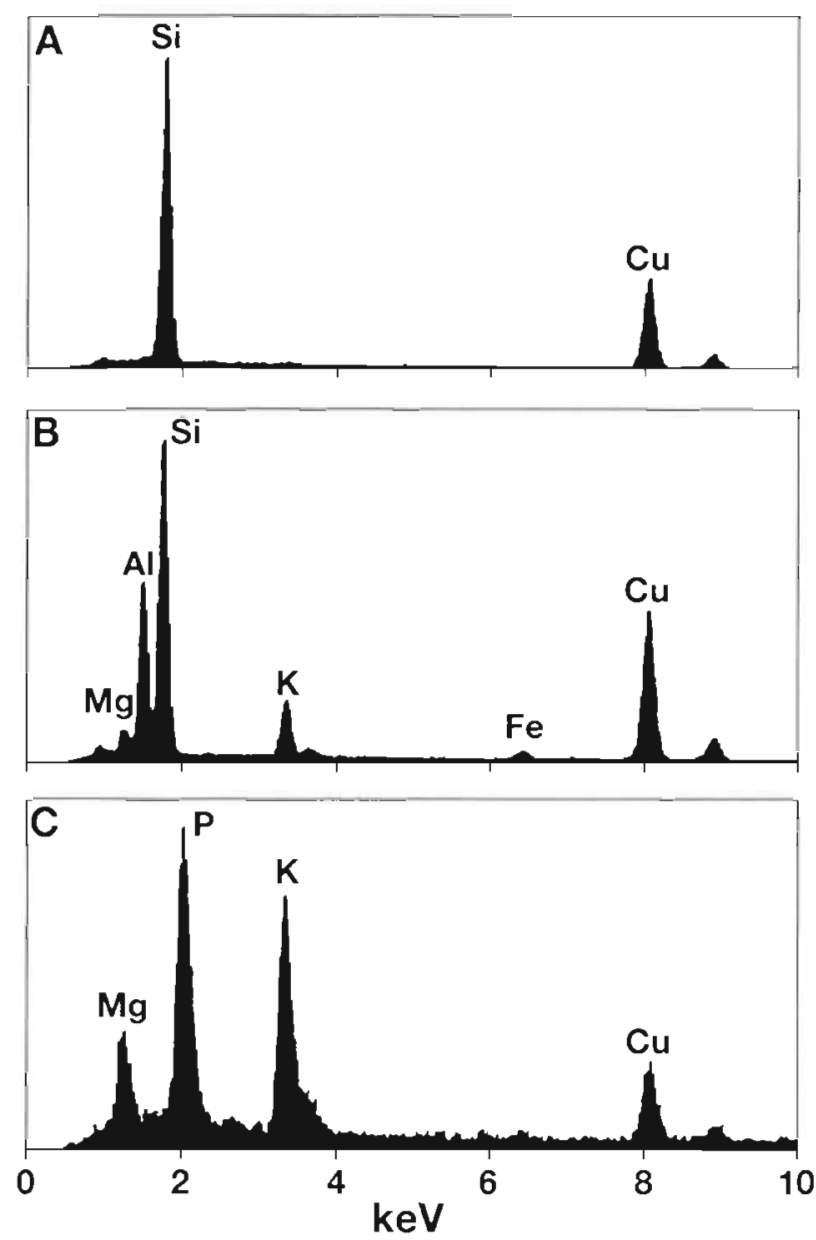

Fig. 5. EDS spectra of 3 commonly found mineral particles within marine snow. (A) Many particles containing silicon as the sole 'heavy' element showed some morphological characteristics of diatom frustules. (B) Many particles containing cilicon and aluminum as the 2 principa! 'heavy' clements also showed a typical clay mineral morphology. (C) Also present was a colloidal phosphorus-rich particle. Copper peaks are from the grids upon which the ultrathin sections were mounted 
Table 1 Comparison of different fixation methods. Only a correlative approach using all methods can reveal information on artifacts and the natural associatrons within marine snow. EDS: energy-dispersive spectrometry

\begin{tabular}{|llll|}
\hline & Glutaraldehyde & Glutaraldehyde + ruthenium red & Nanoplast \\
\hline Fixation of cells & Excellent & Good (grainy) & Poor \\
Fixation of fibrils & Poor & Good & Good \\
Extraction & 50 to $80 \%$ & Minimal & None \\
Resolution & $2.5 \mathrm{~nm}$ & $2.5 \mathrm{~nm}$ & $1 \mathrm{~nm}$ \\
Staining properties & Good & Good & Poor \\
Sample perturbation & Centrifugation & Centrifugation & Minimal \\
& Dehydration & Dehydration & Clear signal \\
EDS & Extraction & Good (tOs and Ru signals) & \\
\hline
\end{tabular}

Elemental composition analyses on a per colloid basis (Leppard 1992a, b) by EDS (Fig. 5) can be done in principle on subjects processed by any of the 3 treatments; the cleanest spectra, however, are derived from Treatment 3.

Treatment 1 is frequently used by marine biologists but produces images of a resolution limit of $-2.5 \mathrm{~nm}$ while Treatment 3 has a resolution limit of $1 \mathrm{~nm}_{\text {; the }}$ limit is set by the grain structure of the resin in the ultrathin sections. Our analysis of the structure of the marine snow matrix at $1 \mathrm{~nm}$ resolution allows us to visualize details of the fibrillar structure, which is not possible with other techniques. Fig. 3A shows that polysaccharide fibrils can act as bridges to connect subcomponents of marine snow particles to each other (e.g. bacteria to mineral particles). The computer reconstruction (Fig. 3B) illustrates how the fibrillar bridges extend into 3 dimensions to bind a microbial community into a heterogeneous multi-component sedimenting unit. Serial sections reveal important details on the morphology of the colloid arrangements in 3 dimensions. Computer reconstructions can be used to combine the different images obtained by the 3 treatments with those from optical microscopy of entire 'snow' particles. A major advantage of Treatment 2 facilitating interpretation is demonstrated here in Fig. 3. When Treatment 1 was applied to an aliquot of the same sample, the extraction (removal) of matrix fibrils during sample processing was so great that most of the fibrils were removed. The conventional Treatment 1 approach would probably not have revealed the sparse fibril network shown in Fig. 3. An independent verification of the fibrillar structure is derived from Treatment 3 (Fig. 4).

The variety in size and morphology of marine fibrils was similar to the variety described for freshwater (Leppard 1986). Long, curved, ribbon-like types were most common and the average diameter was $-5 \mathrm{~nm}$. In contrast to Treatment 2, the fibrils obtained by Treatment 1 did not stain as intensively with the counter-stains (uranyl and lead). The extraction arti- fact found with Treatment 1 might have caused selective removal of the otherwise common fibril types (Leppard 1986). The fibrils obtained from Treatment 3 corresponded in size and morphology to the images of Treatment 2, with 1 exception. The sharply defined ribbons (Leppard 1986) common in images of Treatment 2 were much less common in images from Treatment 3 . The most common morphotype detectable by

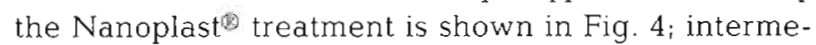
diate morphotypes between those shown in Fig. 4 and those of well-defined ribbon-like fibrils were also found.

The multi-treatment, correlative approach is time consuming and costly when applied to such heterogeneous particles as marine snow. The approach presented herein, however, allowed unsurpassed resolution as compared to scanning electron microscopy techniques and their required harsh preparatory and dehydration steps (Herndl \& Peduzzi 1988). The correlative use of TEM preparatory techniques in conjunction with whole-sample information from optical microscopy allowed us to systematically detect, assess and minimize possible artifacts caused by extraction and dehydration (Table 1). The fact that shrinkage by Treatment 2 is minimal allowed comparisons of glutaraldehyde-based images with images from the Nanoplast ${ }^{\circledR}$ embedding technique as outlined in Treatment 3. Extraction and dehydration artifacts with regard to aquatic colloids and biologically derived flocculent material have recently been reviewed (Leppard 1992a, 1993).

Since an increase in resolution allows us to elucidate better the ultrastructure of colloids and their associations, the proper preservation of the fibrils in their original position is important (Leppard 1992a, 1993). Thus, specific attention was paid in this study to determine preservation artifacts by comparing different approaches. The improved resolution of the morphology of the fibril component of marine snow might also explain the mismatch of the fibril morphotypes obtained by Treatments 2 and 3 ; this mismatch stems partly from the improved capacity to resolve the elec- 
tron-opaque stains used in the visualization and partly from the fact that fibrils embedded in Nanoplast $\left.{ }^{(}\right)$are less perturbed with regard to replacement of water.

In summary, the technology described here to establish the general ultrastructural characteristics of marine snow is applicable to a wide range of hydrated particulate matter. Although this technology requires extreme care, it permits correlation of visualized characteristics so as to detect, assess and minimize artifacts of extraction and shrinkage. It has permitted herein the first large-scale systematic analysis of marine snow matrices at 1 to $3 \mathrm{~nm}$ resolution. We have shown that fibrils constitute the structural framework of the matrix. The next step is to determine the ecophysiological significance of the 3 to $10 \mathrm{~nm}$ fibrils to the matrix framework and to microbial associations. Adjustments of the protocol might be necessary for different types of samples and to improve the costeffectiveness ratio. In the companion paper (Heissenberger et al. 1996, this issue), using the techniques outlined here, we will visualize the microenvironment of the organisms embedded in the fibrillar network of the marine snow matrix and the structure of the bacterial capsule.

Acknowledgements. We are grateful to the staff of the Center for Marine Research (Rovinj, Croatia) and the captain and crew of the RV 'Vila Velebita' for their hospitality and help during sampling and S. Puskaric for the use of his microscope. We thank M. Agis and G. Müller-Nıklas (University of Vienna) for diving assistance, M. M. West, D. T. Flannigan, J. Carson and C. Zorn (Hamilton, Canada) for electron microscope and computer technical services, and J. N. A. Lott [McMaster University) for providing tlectron microscope facilities. Financial support was provided by the National Water Research Institute of Environment Canada to G.G.L. and by grants from the Austrian Science Foundation (FWFgrants 8608 and 9388) to G.J.H. A portion of this work was sponsored by a grant of the New Energy and Industrial Technology Development Organization (NEDO)/Research Institute of Innovative Technology for the Earth (RITE), both of Japan, to G.G.L.

\section{LJTERATURE CITED}

Alldredge A.L, Silver MW (1988) Characteristics, dynamics and signiticance of marine snow. Prog Oceanogr 20:41-82

Aspinall GO (ed) (1982) The polysaccharides, Academic Press, New York

Aspinall GO (1983) Classification of polysaccharides. In Aspinall GO (ed) The polysaccharides. Academic Press, New York, p 1-9

Bachhuber K, Frosch D (1983) Melamine resins, a new class of water-soluble embedding media for electron microscopy. J Microsc 130:1-9

Burnison BK, Leppard GG $\{1933$ isviaiion of coliondai tıbrils from lake water by physical separation techniques. Can $J$ Fish Aquat Sci 40:373-381

Cagle GD, Pfister RM, Vela GR (1972) Improved staining of extracellular polymer for electron microscopy: examination of Azotobacter, Zoogloea, Leuconostoc, and Bacillus Appl Microbiol 24:477-487

Chandler JA (1977) X-ray microanalysis in the electron microscope. North-Holland, Amsterdam

Decho AW, Herndl GJ (1995) Microbial activities and the transformation of organic matter within mucilaginous material. Sci Total Environ 165:32-42

Filella M, Buffle J, Leppard GG (1993) Characterization of submicrometer colloids in freshwaters: evidence for their bridging by organic structures. Wat Sci Tech 27:91-102

Frosch D. Westphal C (1989) Melamine resins and their application in electron microscopy. Electron Microsc Rev 2 $231-255$

Hayat MA. (1981) Fixation for electron microscopy. Academic Press, New York

Heissenberger A, Leppard GG, Herndl GJ (1996) Ultrastructure of marine snow. IL. Microbiological considerations Mar Ecol Prog Ser 135:299-308

Herndl GJ, Peduzzi P (1988) Ecology of amorphous aggregations (marine snow) in the Northern Adriatic Sea: I General considerations. PSZN I: Mar Ecol 9:79-90

Karner M. Fuks D. Herndl GJ (1992) Bacterial activity along a trophic gradient. Microb Ecol 24:243-257

Kenne L, Lindberg B (1983) Bacterial polysaccharides. In Aspinall GO (ed) The polysaccharides. Academic Press New York, p 287-365

Kloareg B, Quatrano RS (1988) Structure of the cell walls of marine algae and ecophysiological function of the matrix polysaccharides. Oceanogr Mar Biol A Rev 26:259-315

Leppard GG (1986) The fibrillar matrix component of lacustrine biofilms. Wat Res 20:697-702

Leppard GG (1992a) Evaluation of electron microscope techniques for the description of aquatic colloids. In: Buffle J, van Leeuwen HP (eds) Environmental particles, Vol. 1 Lewis Publishers, Boca Raton, p 231-289

Leppard GG (1992b) Size, morphology and composition of particulates in aquatic ecosystems: solving speciation problems by correlative electron microscopy. Analyst 117 $595-603$

Leppard GG (1993) Organic flocs in surface waters: their native state and aggregation behavior in relation to contaminant dispersion. In: Rao SS (ed) Particulate matter and aquatic contaminants. Lewis Publishers, Boca Raton. p $169-195$

Leppard GG (1995) The charactenzation of algal and microbial mucilages and their aggregates in aquatic ecosystems. Sci Total Environ 165:103-131

Leppard GG, Burnison BK, Buffle J (1990) Transmission electron microscopy of the natural organic matter of surface waters. Analyt Chim Acta 232:107-121

Leppard GG, Massalski A, Lean DRS (1977) Electron-opaque microscopic fibrils in lakes: their demonstration, their biological derivation and their potential significance in the redistribution of cations. Protoplasma 92:289-309

Lewis PR, Knight DP (1977) Staining methods for sectıoned material. North-Holland, Amsterdam

Lindberg B (1981) Structural studies of polysaccharidesHaworth memorial lecture. Chem Soc Rev 10:409-434

Liss SN, Droppo IG, Flannigan DT, Leppard GG (in press) Floc architecture in wastewater and natural riverine systems. Environ Sci Technol

Luft JH (1971) Ruthenium red and ruthenium violet. I. Chemistry, purification, methods of use for electron microscopy, and mechanisms of action. Anat Rec 171:347-368

Mavrocordatos D, Lienemann CP, Perret D (1994) Energy filtered transmisson electron microscopy for the physico- 
chemical characterization of aquatic submicron colloids. Mikrochim Acta 117:39-47

Painter TJ (1983) Algal polysaccharides. In: Aspinall GO (ed) The polysaccharides. Academic Press, New York, p 195-285

Percival E, McDowell RH (1967) Chemistry and enzymology of marine algal polysaccharides. Academic Press, London

Perret D, Leppard GG, Müller M, Belzile N, DeVitre R, Buffle J (1991) Electron microscopy of aquatic collolds: nonperturbing preparation of specimens in the field. Wat Res 25:1333-1343

Rees DA, Morris ER, Thom D, Madden JK (1982) Shapes and interactions of carbohydrate chains. In: Aspinall GO (ed) The polysaccharides. Academic Press, New York, p 195-290

Reynolds ES (1963) The use of lead citrate at high $\mathrm{pH}$ as

This article was submitted to the editor an electron-opaque stain in electron microscopy. J Cell Biol 17:208-212

Spurr AR (1969) A low-viscosity epoxy resin embedding medium for electron microscopy. J Ultrastruct Res 26: $31-43$

Strycek T, Acreman J, Kerry A, Leppard GG, Nermut MV, Kushner DJ (1992) Extracellular fibril production by freshwater algae and cyanobacteria. Microb Ecol 23: $53-74$

Suzuki N, Kato K (1953) Studies on suspended materials. Marine snow in the sea. Part I. Sources of marine snow. Bull Fac Fish Hokkaido Univ 4:132-135

van Boekel WHM (1992) Phaeocystis colony mucus components and the importance of calcium ions for colony stability. Mar Ecol Prog Ser 87:301-305

Whitfield C (1988) Bacterial extracellular polysaccharides. Can J Microbiol 34:415-420

Manuscript first received: July 6, 1995

Revised version accepted: December 11, 1995 